Irish Math. Soc. Bulletin

Number 69, Summer 2012, 33-46

ISSN 0791-5578

\title{
SPECTRAL PERMANENCE
}

\author{
ROBIN HARTE
}

\begin{abstract}
Several kinds of generalized inverse bounce off one another in the proof of a variant of spectral permanence for $\mathrm{C}^{*}$ embeddings.
\end{abstract}

This represents an expanded version of our talk to the IMS meeting of August 2012, which in turn was based on the work [3] of Dragan Djordjevic and Szezena Zivkovic of Nis, in Serbia.

\section{GelFAND PROPERTY}

Spectral permanence, for $\mathrm{C}^{*}$ algebras, says that the spectrum of an element $a \in A \subseteq B$ of a $\mathrm{C}^{*}$ algebra is the same whether it is taken relative to the subalgebra $A$ or the whole algebra $B$ : this discussion is sparked by the effort to prove that the same is true of a variant of spectral permanence in which the two-sided inverse, whose presence or not defines "spectrum", is replaced by a generalized inverse. The argument involves a circuitous tour through "group inverses", "Koliha-Drazin inverses" and "Moore-Penrose inverses"; it turns out that the induced variants of spectral permanence are curiously inter-related.

Suppose $T: A \rightarrow B$ is a semigroup homomorphism, where we insist that a semigroup $A$ has an identity 1 , and that a homomorphism $T: A \rightarrow B$ respects that: we might indeed talk about a functor between categories. It then follows, writing $A^{-1}$ for the invertible group in $A$, that

$$
T\left(A^{-1}\right) \subseteq B^{-1},
$$

or equivalently, turning it inside out,

$$
A^{-1} \subseteq T^{-1} B^{-1} .
$$

2010 Mathematics Subject Classification. 46H05, 47A05, 47A53.

Key words and phrases. Semigroup homomorphisms, spectral permanence, generalized permanence, simply polar elements.

Received on 10-3-2011; revised 5-8-2012. 
At its most abstract then "spectral permanence" for the homomorphism $T$ says that (1.2) holds with equality:

$$
T^{-1} B^{-1} \subseteq A^{-1} .
$$

In words, it is tempting to describe (1.3) by saying "Fredholm implies invertible". We shall also describe (1.3) as the Gelfand property, since it also holds, famously, when

$$
T=\Gamma: A \rightarrow C(X) \subseteq \mathbf{C}^{X}
$$

is the Gelfand representation of a commutative Banach algebra $A$; here of course $X=\sigma(A)$ is the "maximal ideal space" of the algebra $A$. We might notice a secondary instance of spectral permanence in the embedding

$$
C(X) \subseteq \mathbf{C}^{X}
$$

of continuous functions among arbitrary functions; similarly, for a Banach space $X$, the embedding

$$
B(X) \subseteq L(X)
$$

of bounded operators among arbitrary linear operators has spectral permanence, but only thanks to the ministrations of the open mapping theorem. Another elementary example is the left regular representation

$$
L: A \rightarrow A^{A}
$$

of the semigroup $A$ as mappings, where, for $a \in A$,

$$
L_{a}(x)=a x(x \in A) .
$$

Less familiar is a commutant embedding

$$
J: A=\operatorname{comm}_{B}(K) \rightarrow B,
$$

where

$$
\operatorname{comm}_{B}(K)=\{b \in B: a \in K \Longrightarrow b a=a b\}
$$

and of course $J(a)=a$ : here spectral permanence reflects the fact that two-sided inverses double commute:

$$
a \in B^{-1} \Longrightarrow a^{-1} \in \operatorname{comm}_{B}^{2}(a) .
$$

If in particular the semigroup $A$ is a ring, having therefore a background "addition" and a distributive law, then we can quotient out the Jacobson radical

$$
\operatorname{Rad}(A)=\left\{a \in A: 1-A a \subseteq A^{-1}\right\},
$$


in which every possible expression $1-c a$ has an inverse: now it is easily checked that

$$
K: a \mapsto a+\operatorname{Rad}(A)(A \rightarrow A / \operatorname{Rad}(A))
$$

has spectral permanence. Our final example will be the most familiar, if not by any means the most elementary: it is the determinant

$$
\operatorname{det}: \mathbf{C}^{n \times n} \rightarrow \mathbf{C},
$$

which indeed "determines" whether or not a square matrix is invertible.

\section{Spectral PeRmanence}

Mathematicians are thus prepared to go to a lot of trouble to establish spectral permanence. If we specialise to linear homomorphisms between (complex) linear algebras then we meet the phenomenon of spectrum, defining for each $a \in A$,

$$
\sigma_{A}(a)=\left\{\lambda \in \mathbf{C}: a-\lambda \notin A^{-1}\right\} ;
$$

the idea is to harness complex analysis to the theory of invertibility. Now we can rewrite (1.1) to say that, for arbitrary $a \in A$,

$$
\sigma_{B}(T a) \subseteq \sigma_{A}(a)
$$

while the Gelfand property (1.3) says that (2.2) holds with equality, giving indeed "spectral permanence".

If we specialise to isometric Banach algebra homomorphisms then there is built in a certain degree of spectral permanence, to the extent that we always get

$$
\partial \sigma_{A}(a) \subseteq \sigma_{B}(T a):
$$

the topological boundary of the larger spectrum is included in the smaller. Equivalently, it turns out, this means that

$$
\sigma_{A}(a) \subseteq \eta \sigma_{B}(T a),
$$

where the connected hull $\eta K$ of a compact subset $K \subseteq \mathbf{C}$ is the complement of the unbounded connected component of the complement $\mathbf{C} \backslash K$. This has spin-off: if for a particular element $a \in A$ either the larger spectrum is all boundary,

$$
\sigma_{A}(a) \subseteq \partial \sigma_{A}(a),
$$

or the smaller spectrum fills out its connected hull,

$$
\eta \sigma_{B}(T a) \subseteq \sigma_{B}(T a),
$$


then the homomorphism $T: A \rightarrow B$ has "spectral permanence at" $a \in A$, in the sense of equality in (2.2). This holds if for example the spectrum is either real or finite.

If more generally the homomorphism $T: A \rightarrow B$ is one-one there is at least inclusion

$$
\text { iso } \sigma_{A}(a) \subseteq \sigma_{B}(T a)
$$

\section{Generalized permanence}

If $A$ is a semigroup we shall write

$$
A^{\cap}=\{a \in A: a \in a A a\}
$$

for the "regular" or relatively regular elements of $A$, those $a \in A$ which have a generalized inverse $c \in A$ for which

$$
a=a c a \text { : }
$$

we remark that if (3.2) holds the products

$$
p=c a=p^{2}, q=a c=q^{2}
$$

are both idempotent. Generally if $T: A \rightarrow B$ is a homomorphism there is inclusion

$$
T\left(A^{\cap}\right) \subseteq B^{\cap} \subseteq B
$$

and hence also

$$
A^{\cap} \subseteq T^{-1}\left(B^{\cap}\right) \subseteq A .
$$

If there is equality in (3.4) we shall say that $T$ has generalized permanence. This happens for example when

$$
T^{-1}(0) \subseteq A^{\cap}, T(A)=B:
$$

recall the implication

$$
(a-a A a) \cap A^{\cap} \neq \emptyset \Longrightarrow a \in A^{\cap} .
$$

This does not however happen when $T$ is quotienting out the radical as in (1.10), unless the $\operatorname{ring} A$ is semi simple: for notice

$$
\operatorname{Rad}(A) \cap A^{\cap}=\{0\} .
$$

It follows that spectral permanence is not in general sufficient for generalized permanence. Indeed by (3.8) spectral and generalized permanence together imply that a homomorphism $T: A \rightarrow B$ is one one; further (1.5) shows that spectral permanence and one one do not together imply generalized permanence. If $A$ is the ring of continuous homomorphisms $a: X \rightarrow X$ on a Hausdorff topological 
abelian group $X$ then it is necessary for $a \in A^{\cap}$ that $a$ have closed range

$$
a(X)=\operatorname{cl} a(X):
$$

this is because

$$
a(X)=a c(X)=(1-a c)^{-1}(0)
$$

is the null space of the complementary idempotent. Thus the embedding (1.6) is another example with spectral but not generalized permanence.

\section{Simple PERMANENCE}

If in particular there is $c \in A$ for which

$$
a-a c a=0=a c-c a,
$$

then $a \in A$ is very special; this happens if $a \in A$ is either invertible, or idempotent, or more generally the commuting product of an invertible and an idempotent. When (4.1) holds we shall say that $a \in A$ is simply polar: in Banach-algebra-land $0 \in \mathbf{C}$ can be at worst a simple pole of the resolvent mapping

$$
(z-a)^{-1}: \mathbf{C} \backslash \sigma(a) \rightarrow A .
$$

In the group theory world the product cac is referred to as the group inverse for $a \in A$. We remark that it is necessary and sufficient for $a \in A$ to be simply polar that

$$
a \in a^{2} A \cap A a^{2} \quad:
$$

indeed [15],[19],[20] there is implication

$$
a^{2} u=a=v a^{2} \Longrightarrow a u=v a, a u a=a=a v a,
$$

giving (4.1) with $c=$ vau.

We shall write $\operatorname{SP}(A)$ for the simply polar elements of a semigroup $A$ and observe, for homomorphisms $T: A \rightarrow B$, that

$$
T \mathrm{SP}(A) \subseteq \mathrm{SP}(B) \subseteq B
$$

and hence

$$
\mathrm{SP}(A) \subseteq T^{-1} \mathrm{SP}(B) \subseteq A ;
$$

when there is equality in (4.5) we shall say that $T: A \rightarrow B$ has simple permanence. The counterimage $T^{-1} \mathrm{SP}(B) \subseteq A$ is sometimes known [2],[18],[16] as the "generalized Fredholm" elements of $A$. 
We remark that spectral permanence does not in general, or even together with one-one-ness, imply simple permanence: return to (3.8) and (1.5).

In general

$$
\mathrm{SP}(A) \subseteq A^{\cup} \equiv\left\{a \in A: a \in a A^{-1} a\right\}
$$

and hence

$$
\mathrm{SP}(A) \cap A_{\text {left }}^{-1}=A^{-1}=\mathrm{SP}(A) \cap A_{\text {right }}^{-1} .
$$

This will show again that spectral permanence together with one one is not sufficient for generalized permanence:

Theorem 4.1. If $B_{\text {left }}^{-1} \neq B_{\text {right }}^{-1}$ then there exist $A$ and $T: A \rightarrow B$ for which $T$ is one one with spectral but not generalized permanence.

Proof. If $A$ is commutative then $A^{\cap}=\mathrm{SP}(A)$ and hence

$$
T\left(A^{\cap}\right) \subseteq \mathrm{SP}(B) \subseteq B^{\cap},
$$

and if

$$
T\left(A^{\cap}\right) \cap B_{\text {left }}^{-1} \backslash B^{-1} \neq \emptyset
$$

then $T$ does not have generalized permanence. Thus find $a \in B_{\text {left }}^{-1} \backslash$ $B^{-1}$ and, recalling (1.9), take

$$
T=J: \operatorname{comm}_{B}^{2}(a) \subseteq B
$$

The familiar example is to take $B=L(X)$ to be the linear mappings on the space $X=\mathbf{C}^{\mathbf{N}}$ of all complex sequences and $a \in B$ to be the forward shift. Conversely however simple permanence together with one-one-ness does imply spectral permanence:

Theorem 4.2. For semigroup homomorphisms

one one and simple permanence implies spectral permanence,

while conversely

simple and spectral permanence implies one one.

Proof. The last implication is (3.8); conversely observe

$$
\mathrm{SP}(A) \cap T^{-1} B_{\text {left }}^{-1} \subseteq A^{\cup} \cap T^{-1} B_{\text {left }}^{-1} \subseteq A^{-1}+T^{-1}(0)
$$


When we specialise to rings of mappings then simple polarity is characterized by "ascent" and "descent":

Theorem 4.3. If $A=L(X)$ is the additive, or linear, operators on an abelian group, or vector space, $X$ then necessary and sufficient for $a \in A$ to be simply polar is that it has ascent $\leq 1$,

$$
a^{-2}(0) \subseteq a^{-1}(0) ; \text { equivalently } a^{-1}(0) \cap a(X)=O \equiv\{0\},
$$

and also descent $\leq 1$,

$$
a(X) \subseteq a^{2}(X) ; \text { equivalently } a^{-1}(0)+a(X)=X .
$$

The same characterization is valid when $A=B(X)$ for a Banach space $X$.

Proof. The complementary subspaces $a^{-1}(0)$ and $a(X)$ determine the idempotent $p: X \rightarrow X$, defined by setting

$$
p(\xi) \in a(X) ; \xi-p(\xi) \in a^{-1}(0)
$$

for each $\xi \in X$, whose boundedness, together with the closedness of the range $a(X)$, follows ([7] Theorem 4.8.2) from the open mapping theorem; and finally, if $\xi \in X$,

$$
c(\xi)=c p(\xi) ; c a(\xi)=p(\xi)
$$

We remark that, on incomplete spaces, the conditions (4.5) and (4.6) are not sufficient for simple polarity: indeed it is possible for $a \in B(X)$ to be one one and onto but not in $B(X)^{\cap}$ : the obvious example is the "standard weight" $a=w$ on $X=c_{00} \subseteq c_{0}$ defined by setting

$$
w(\xi)_{n}=(1 / n) \xi_{n} .
$$

Even together with the assumption $a \in A^{\cap}$, however, the conditions (4.5) and (4.6) are ([7] (7.3.6.8)) not sufficient for simple polarity (4.1) when $A=B(X)$ for an incomplete normed space $X$.

\section{DRAZIN PERMANENCE}

More generally if there is $n \in \mathbf{N}$ for which $a^{n}$ is simply polar we shall also say that $a \in A$ is "polar", or Drazin invertible. If $a \in A$ is polar then there is $c \in A$ for which $a c=c a$ and $a-a c a$ is nilpotent. If we further relax this to "quasinilpotent" we reach the condition that $a \in A$ "quasipolar". Specifically if we write

$$
\mathrm{QN}(A)=\left\{a \in A: 1-\mathrm{C} a \subseteq A^{-1}\right\}
$$


for the quasinilpotents of a Banach algebra $A$ then $a \in \mathrm{QN}(A)$ if and only if

$$
\sigma_{A}(a) \subseteq\{0\},
$$

while with some complex analysis we can prove that if $a \in \mathrm{QN}(A)$ then

$$
\left\|a^{n}\right\|^{1 / n} \rightarrow 0(n \rightarrow \infty) .
$$

In the ultimate generalization of "group invertibility", we shall write $\mathrm{QP}(A)$ for the quasipolar elements $a \in A$, those which have a spectral projection $q \in A$ for which ( $\mathrm{cf}[8]$ )

$$
q=q^{2} ; a q=q a ; a+q \in A^{-1} ; a q \in \mathrm{QN}(A) .
$$

Now [17] the spectral projection and the Koliha-Drazin inverse

$$
a^{\bullet}=q, a^{\times}=(a+q)^{-1}(1-q)
$$

are uniquely determined and lie in the double commutant of $a \in A$. It is easy to see that if (5.3) is satisfied then

$$
0 \notin \operatorname{acc} \sigma_{A}(a):
$$

the origin cannot be an accumulation point of the spectrum; conversely if (5.5) holds then we can display the spectral projection as a sort of "vector-valued winding number"

$$
a^{\bullet}=\frac{1}{2 \pi i} \oint_{0}(z-a)^{-1} d z,
$$

where we integrate counter clockwise round a small circle $\gamma$ centre the origin whose connected hull $\eta \gamma$ is a disc whose intersection with the spectrum is at most the point $\{0\}$. Now generally for a homomorphism $T: A \rightarrow B$ there is inclusion

$$
T \mathrm{QP}(A) \subseteq \mathrm{QP}(B),
$$

while if $T: A \rightarrow B$ has spectral permanence in the sense (1.3) then it is clear from (5.5) that there is also "Drazin permanence" in the sense that

$$
\mathrm{QP}(A)=T^{-1} \mathrm{QP}(B) \subseteq A:
$$

Theorem 5.1. For Banach algebra homomorphisms $T: A \rightarrow B$ there is implication

$$
\text { spectral permanence } \Longrightarrow \text { Drazin permanence . }
$$

Proof. Equality in (2.2), together with (5.5) 
The example of Theorem 4.1 also shows that the left regular representation $L: A \rightarrow B(A)$, with $A=B(X)$ for a normed space $X$, does not always have generalized permanence; however we do have a sort of "closed range permanence": there is implication

$$
L_{a} A=\mathrm{cl} L_{a} A \Longrightarrow a(X)=\operatorname{cl} a(X):
$$

indeed if $a \xi_{n} \rightarrow \eta$ and $\varphi \in X^{*}$ and $\varphi(\xi)=1$ then, with $\varphi \odot \eta: \zeta \mapsto$ $\varphi(\zeta) \eta$

$$
L_{a}(\varphi \odot \eta)=L_{a}(b) \Longrightarrow \eta=a(b \xi) .
$$

Generally

Theorem 5.2. If $T: A \rightarrow B$ is arbitrary then

$$
\mathrm{QP}(A) \cap T^{-1}\left(B^{-1}\right) \subseteq A^{-1}+T^{-1}(0)
$$

and if $T: A \rightarrow B$ is one one then

$$
\mathrm{QP}(A) \cap T^{-1} \mathrm{SP}(B)=\mathrm{SP}(A) .
$$

Hence if $a \in B$ and $T=J: A=\operatorname{comm}_{B}^{2}(a) \subseteq B$ then

$$
A^{\cap}=T^{-1} \mathrm{SP}(B) \text {. }
$$

It follows that if $T^{-1}(0)=O$ then

$$
\text { Drazin } \Longrightarrow \text { simple } \Longrightarrow \text { spectral permanence . }
$$

Proof. Uniqueness guarantees that the spectral projection $T(a)^{\bullet}$ of $T a \in \mathrm{SP}(B) \subseteq \mathrm{QP}(B)$ commutes with $T(a) \in B$, and one-one-ness guarantees the same for $a \in A$

For Banach algebra homomorphisms therefore there is an improved version of Theorem 4.2, of the three conditions

$$
\text { spectral permanence ; simple permanence ; one one, }
$$

any two imply the third.

If we rework Theorem 4.1 with $B=B\left(\ell_{2}\right)$ then it is clear that isometric homomorphisms with spectral permanence need not have generalized permanence: indeed the forward shift $a=u \in B^{\cap} \backslash$ $\mathrm{QP}(A)$ is not even quasipolar: we recall that the spectrum of $u$ is the closed unit disc, violating (5.5).

Theorem 4.1 was obtained in this way ([3] Theorem 3.2) in [3]. Of course (cf [9],[17]) "quasinilpotents" and "quasipolars" are only available in Banach algebras; Theorem 4.1 above, using "simply polar" elements, is conceptually much simpler. 


\section{Moore-Penrose Permanence}

We recall that a "C* algebra" is a Banach algebra which also has an involution $a \mapsto a^{*}$ which is conjugate linear, reverses multiplication, respects the identity and satisfies the "B* condition"

$$
\left\|a^{*} a\right\|=\|a\|^{2}(a \in A) .
$$

Historically the term " $\mathrm{C}^{*}$ algebra" was reserved for closed ${ }^{*}$-subalgebras of the algebras $B(X)$ for Hilbert spaces $X$; however the Gelfand-Naimark-Segal (GNS) representation

$$
\Gamma: A \rightarrow B\left(\Xi_{A}\right)
$$

takes an arbitrary "B* algebra" $A$ isometrically into the algebra of operators on a rather large Hilbert space $\Xi_{A}$ built from its "states": a defect of (6.2) would be that if already $A=B(X)$ we do not get back $\Xi_{A}=X$. In the opinion of this writer these terms " $\mathrm{B}^{*}$ algebra" and " $\mathrm{C}^{*}$ algebra" could easily ([7] Chapter 8$)$ have been Hilbert algebra. When in particular $A=B(X)$ for a Hilbert space $X$ then the closed range condition (3.9) is sufficient for relative regularity $a \in A^{\cap}$ : indeed we can satisfy $(2.2)$ by setting

$$
c(\xi)=c(q \xi) ; c(a \xi)=p(\xi)(\xi \in X),
$$

where $q^{*}=q=q^{2}$ and $p^{*}=p=p^{2}$ are the orthogonal projections on the range $a(X)$ and the orthogonal complement $a^{-1}(0)^{\perp}$ of the null space. The element $c \in A$ given by (6.3) satisfies four conditions:

$$
a=a c a ; c=c a c ;(c a)^{*}=c a ;(a c)^{*}=a c,
$$

and is known as the Moore-Penrose inverse of $a \in B(X)$ : more generally in a $C^{*}$ algebra $A$ the conditions (6.4) uniquely determine at most one element

$$
c=a^{\dagger} \in A,
$$

lying ([11] Theorem 5) in the double commutant of $\left\{a, a^{*}\right\}$, and still known as a "Moore-Penrose inverse" for $a \in A$. Now it is a result of Harte and Mbekhta ([11] Theorem 6) that generally there is equality

$$
A^{\cap}=A^{\dagger} \text { : }
$$

in an arbitrary $\mathrm{C}^{*}$ algebra, every relatively regular element has a Moore Penrose inverse. The argument, and a slight generalization, proceeds with the aid of the Drazin inverse.

More generally, on a semigroup $A$, an involution $a \mapsto a^{*}$ satisfies

$$
\left(a^{*}\right)^{*}=a ;(c a)^{*}=a^{*} c^{*} ; 1^{*}=1 .
$$


In rings and algebras we also ask that the involution be additive, or conjugate linear. The $\mathrm{B}^{*}$ condition (6.7) implies that, for arbitrary $a, x \in A$,

$$
\|a x\|^{2} \leq\left\|x^{*}\right\|\left\|a^{*} a x\right\|,
$$

which in turn gives cancellation

$$
L_{a^{*} a}^{-1}(0) \subseteq L_{a}^{-1}(0) .
$$

Generally the hermitian or "real" elements of $A$ are given by

$$
\operatorname{Re}(A)=\left\{a \in A: a^{*}=a\right\} .
$$

The Moore-Penrose inverse $a^{\dagger}$ of (6.4), if it exists, is unique and double commutes with $a$ and $a^{*}$. We pause to notice the star polar elements of a semigroup $A$ :

$$
\mathrm{SP}^{*}(A)=\left\{a \in A: a^{*} a \in A^{\cap}\right\} ;
$$

now we claim

Theorem 6.1. If the involution * on the semigroup $A$ is cancellable then

$$
A^{\dagger} \subseteq \operatorname{SP}^{*}(A) \subseteq A^{\cap} .
$$

Proof. With cancellation there is implication

$$
a \in \operatorname{SP}^{*}(A) \Longrightarrow a \in a A a^{*} a \subseteq A a^{*} a \cap a A a,
$$

and equality

$$
\operatorname{Re}(A) \cap \mathrm{SP}^{*}(A)=\operatorname{Re}(A) \cap \operatorname{SP}(A),
$$

If $a=a c a$ with $c=a^{\dagger}$ then

$$
a^{*} a=a^{*}(a c)(a c)^{*} a=a^{*} a c c^{*} a^{*} a \in a^{*} a A a^{*} a:
$$

conversely, by cancellation,

$$
a^{*} a=a d a^{*} a \Longrightarrow a=a d a^{*} a \quad:
$$

hence also

$$
a \in A a^{*} a ; \Longleftrightarrow a^{*} \in a^{*} a A .
$$

Hence if $a^{*}=a$ then (4.2) follows

It is now clear that an isometric $\mathrm{C}^{*}$ homomorphism has "MoorePenrose permanence":

Theorem 6.2. If $T: A \rightarrow B$ has simple permanence then

$$
T^{-1} B^{\dagger} \subseteq A^{\dagger} \text {. }
$$


Proof. We claim

$$
A^{\dagger}=\left\{a \in A: a^{*} a \in \mathrm{SP}(A)\right\},
$$

with implication

$$
a^{*} a \in \mathrm{SP}(A) \Longrightarrow a^{\dagger}=\left(a^{*} a\right)^{\times} a^{*} .
$$

If $a \in A^{\dagger}$ with $a=a c a$ and $(c a)^{*}=c a$ then, with $d=c c^{*}$, we have

$$
a^{*} a d=a^{*} a c c^{*}=a^{*} c^{*}=a^{*} c^{*} a^{*} c^{*}=c a
$$

and

$$
d a^{*} a=c c^{*} a^{*} a=c a .
$$

Conversely if $a^{*} a=a^{*} a d a^{*} a$ with $a^{*} a d=d a^{*} a$ with (wlog) $d=d^{*}$ then, using cancellation, with $c=d a^{*}$,

$$
a c a=a d a^{*} a=a \text { and } c a=d a^{*} a=a^{*} a d=a^{*} c^{*} .
$$

Now if $a \in A$ there is implication

$$
T a \in B^{\dagger} \Longrightarrow T\left(a^{*} a\right) \in \mathrm{SP}(B) \Longrightarrow a^{*} a \in \mathrm{SP}(A) \Longrightarrow a \in A^{\dagger}
$$

Our main result is a slight generalization, and a new proof, of the Harte/Mbekhta result (6.6), and at the same time "generalized permanence", equality in (3.4), for isometric $\mathrm{C}^{*}$ homomorphisms. One way to go, thanks to the Gelfand/Naimark/Segal representation, is to look first in the very special algebra $D=B(X)$ of bounded Hilbert space operators:

Theorem 6.3. If $d \in D=B(X)$ for a Hilbert space $X$ then

$$
\left(d^{*} d\right)^{-1}(0) \subseteq d^{-1}(0)
$$

and

$$
\operatorname{cl} d(X)+d^{*-1}(0)=X ;
$$

hence if $\operatorname{cl} d(X)=d(X)$ then

$$
d^{*}(X)=d^{*} d(X), \text { andcl } d^{*} d(X)=d^{*} d(X) .
$$

There is inclusion

$$
\operatorname{Re}(D) \cap D^{\cap} \subseteq \mathrm{SP}(D)
$$

hence

$$
d \in D^{\cap} \Longrightarrow d \in \operatorname{SP}^{*}(D) \Longrightarrow d^{*} d \in \operatorname{SP}(D) \Longrightarrow d \in D^{\dagger} .
$$


Proof. For arbitrary $\xi \in X$ there is [3] inequality

$$
\|d \xi\|^{2} \leq\|\xi\|\left\|d^{*} d \xi\right\|
$$

and also

$$
\operatorname{cl} d(X)=d^{*-1}(0)^{\perp}
$$

Both of the Harte/Mbekhta observations now follow:

Theorem 6.4. If $T: A \rightarrow B$ is isometric then

$$
T^{-1}\left(B^{\cap}\right) \subseteq A^{\dagger} .
$$

Proof. With $S: B \rightarrow D=B(X)$ a GNS mapping we argue, using again Theorem 4.2 , together with "spectral permanence at" $a^{*} a$ (which has of course real spectrum),

$$
T a \in B^{\cap} \Longrightarrow S T\left(a^{*} a\right) \in \mathrm{SP}(D) \Longrightarrow a^{*} a \in \mathrm{SP}(A) \Longrightarrow a \in A^{\dagger}
$$

In the situation of (6.14),

$$
a=a^{*} \in A^{\cap} \Longrightarrow a^{\dagger}=a^{\times} ; 1-a^{\dagger} a=a^{\bullet} .
$$

Theorem 6.4 has an obvious extension to homomorphisms with closed range:

Theorem 6.5. If $T: A \rightarrow B$ has closed range then there is implication, for arbitrary $a \in A$,

$$
T(a) \in B^{\cap} \Longrightarrow a+T^{-1}(0) \in\left(A / T^{-1}(0)\right)^{\cap} .
$$

Proof. Apply Theorem 6.4 to the bounded below $T^{\wedge}: A / T^{-1}(0) \rightarrow$ $B$

\section{REFERENCES}

[1] SR Caradus, Generalized inverses and operator theory, Queens papers Pure Appl. Math. Ontario 50 (1978)

[2] SR Caradus, WE Pfaffenberg and B Yood, Calkin algebras and algebras of operators on Banach spaces, Dekker 1974.

[3] DS Djordjević, S Zivković-Zlatanović and RE Harte, Spectral permanence and the Moore-Penrose inverse, Proc. Amer. Math. Soc. 140 (2012) 8237-45.

[4] DS Djordjević, JJ Koliha and I Straskraba, Factorization of EP elements of $C^{*}$ algebras, Linear Multilinear Alg. 57 (2009) 587-594.

[5] DS Djordjević and V Rakocević, Lectures on generalized inverses, University of Nis 2008.

[6] JJ Grobler and H Raubenheimer, Spectral properties of elements of different Banach algebras, Glasgow Math. Jour. 33 (1991) 11-20. 
[7] RE Harte, Invertibility and singularity, Dekker 1988.

[8] RE Harte Spectral projections, Irish Math. Soc. Newsletter 14 (1984) 10-15.

[9] RE Harte, On quasinilpotents in rings, PanAmerican Jour. Math. 1 (1991) $10-16$.

[10] RE Harte, Unspectral sets, Rendiconti Mat. 56 (1998) 69-77.

[11] RE Harte and M Mbekhta, On generalized inverses in $C^{*}$ algebras, Studia Math. 103 (1992) 71-77.

[12] RE Harte and M Mbekhta, On generalized inverses in $C^{*}$ algebras II, Studia Math 106 (1993) 129-138.

[13] RE Harte and M O'Searcoid, Positive elements and the $B^{*}$ condition, Math. Zeit. 193 (1986) 1-9.

[14] RE Harte and AW Wickstead, Boundaries, hulls and spectral mapping theorems, Proc. Royal Irish Acad. 81A (1981) 201-208.

[15] R Hartwig and J Lu, On the group structure of unit regular ring elements, Pacific Jour. Math. 71 (1977).

[16] G Kantun-Montiel, SV Djordjević and RE Harte, On semigroup inverses, Funct. Anal. Approx. Comp. 1 (2009) 11-18.

[17] JJ Koliha, A generalized Drazin inverse, Glasgow Math. Jour. 38 (1996) 367-381.

[18] Ch Schmoeger, On a class of generalized Fredholm operators I-VII, Demonstratio Math. 30-33 (1997-2000).

[19] M Xavier, On the converse of a theorem of Harte and Mbekhta, Studia Math. 184 (2008) 149-152.

[20] M Xavier, On generalized inverses and Green's relations, Linear Alg. Appl. 434 (2011) 1830-1844.

Robin Harte taught for twenty years at University College, Cork, and retired a long time ago, but somehow never goes away: his ghost can sometimes be seen at the TCD-UCD Analysis Seminar.

School of Mathematics, Trinity College Dublin

E-mail address: rharte@maths.tcd.ie 